\title{
The Number of Monotone and Self-Dual Boolean Functions ${ }^{1}$
}

\author{
L. HAVIAROVA AND E. TOMAN
}

\begin{abstract}
In the present paper we study properties of pre-complete class of Boolean functions - monotone Boolean functions. We discuss interval graph, the abbreviated d.n.f., a minimal d.n.f. and a shortest d.n.f. of this function. Then we present a d.n.f. with the highest number of conjunctionsand we determinate the exact number of them. We count the number of monotone Boolean functions with some special properties. In the end we estimate the number of Boolean functionthat are monotone and self-dual at the same time.
\end{abstract}

Mathematics Subject Classification 2000: 05C80 (Random graphs), 60C05 Combinatorial probability), 68R10 (Graph theory), 06E30 (Boolean function)

Additional Key Words and Phrases: Monotone Boolean function, self-dual Boolean function, disjunctive normal form, interval graph

\section{INTRODUCTION}

It is well-known that the free Boolean algebra on $n$ free generators is isomorphic to the Boolean algebra of Boolean functions of $n$ variables. The free bounded distributive lattice on $n$ free generators is isomorphic to the bounded lattice of monotone Boolean functions of $n$ variables [12], [25], [26]. For modern applications of monotone Boolean functions see [27].

A Boolean function can be represented by several types of graphs. Among them, the greatest attention has been devoted to the study of the graph $G(f)$ induced by the vertices of the $n$-cube, on which the Boolean function $f$ takes the value 1 . This geometric representation was introduced by Yablonskiy [1]. The concept of the interval graph of a Boolean function was defined by Sapozhenko [5]. The interval graph is a graph associated with a Boolean function $f$ such that the vertices correspond to maximal intervals of $f$ and two vertices are joined with an edge if the intersection of the corresponding intervals is nonempty.

\footnotetext{
${ }^{1}$ Supported by VEGA grant No 1/1005/12
} 
The problem about the number of monotone Boolean function of $n$ variables $\Psi(n)$ was presented by R. Dedekind in 1897 [12] and he solved this problem for $n \leq 4$. R. Church in 1940 [13] and M. Ward in 1945 [14] solved it for $n=5$ and $n=6$ respectively. M. Ward proved that $\Psi(n)>2^{\left(\begin{array}{c}n \\ {[n / 2]}\end{array}\right)}$. In 1945 E. N. Gilbert showed that $\Psi(n)<n^{\left(\begin{array}{c}n \\ n / 2\end{array}\right)}+2$ [15]. K. Yamamoto presented that $\Psi(n)$ is even for an arbitrary even $n[16]$ and proved that

$$
\log _{2} \Psi(n)<\left(\begin{array}{c}
n \\
{[n / 2]}
\end{array}\right) \cdot\left(1+O\left(n^{-1}\right)\right) \cdot \log _{2} \sqrt{\frac{\pi \cdot n}{2}}
$$

for an arbitrary $n$ [20]. V. K. Korobkov showed that $\Psi(n)<2^{4,21 .\left(\left[\begin{array}{c}n \\ {[n / 2]}\end{array}\right)\right.}$ and later G. Hansel reduced this estimate and obtained $\Psi(n)<3^{\left(\begin{array}{c}n \\ {[n / 2]}\end{array}\right)}$ [17]. D. Kleitman provided new upper bound for $\Psi(n)$ [22],

$$
\Psi(n) \leq 2^{\left(1+\beta_{n}\right) \cdot\left(\begin{array}{c}
n \\
{[n / 2]}
\end{array}\right),}
$$

where $\beta_{n}=\frac{e, \ln n}{\sqrt{n}}$. This together with the inequality $\Psi(n)>2^{\left(\begin{array}{c}n \\ {[n / 2]}\end{array}\right)}$ provides an asymptotic estimate for $\log _{2} \Psi(n)$

$$
\log _{2} \Psi(n) \sim\left(\begin{array}{c}
n \\
{[n / 2]}
\end{array}\right)
$$

Kleitman and Markowsky in [18] and [19] gained more precise solution for the upper and the lower bound of $\Psi(n)$

$$
2^{\left(1+\alpha_{n}\right) \cdot\left(\begin{array}{c}
n \\
{[n / 2]}
\end{array}\right)} \leq \Psi(n) \leq 2^{\left(1+\dot{\beta}_{n}\right) \cdot\left(\begin{array}{c}
n \\
{[n / 2]}
\end{array}\right)}
$$

where $\alpha=e .2^{\frac{-n}{2}}$ a $\beta^{\prime}{ }_{n}=\frac{e ́ . l n n}{n}$. A. D. Korshunov [21] presented the best solution so far, an asymptotic estimate of $\Psi(n)$, for even $n \rightarrow \infty$

$$
\Psi(n) \sim 2\left(\begin{array}{c}
n \\
{\left[\frac{n}{2}\right]}
\end{array}\right) \exp \left(\left(\frac{n}{2}-1\right)\left(\frac{1}{2^{\frac{n}{2}}}+\frac{n^{2}}{2^{n+5}}-\frac{n}{2^{n+4}}\right)\right)
$$

and for odd $n \rightarrow \infty$ 


$$
\begin{aligned}
\left.\Psi(n) \sim 2.2^{\left(\frac{n-1}{2}\right)}\right) & \exp \left(\left(\frac{n-3}{2}\right)\left(\frac{1}{2^{\frac{n+3}{2}}}+\frac{n^{2}}{2^{n+6}}-\frac{n}{2^{n+3}}\right)\right. \\
+ & \left.\left(\frac{n-1}{2}\right)\left(\frac{1}{2^{\frac{n+1}{2}}}+\frac{n^{2}}{2^{n+4}}\right)\right) .
\end{aligned}
$$

S. V. Yablonskiy showed that d.n.f. of a monotone Boolean function does not contain negated variables [2].

Self-dual Boolean functions was defined by E. Postom [23]. Fredman and Khachiyan (1996), Gaur and Krishnamurti (2008) showed the following: the monotone Boolean function $f$ is self-dual if for the number of disjunctions $k$ of the abbreviated d.n.f. of $f$ holds $k \leq n^{2}$. M. Altun and M. D. Riedel inproved this result [24]. They proved that $f$ is self-dual if $k \leq n$. They also investigated monotone and at the same time self-dual Boolean function with the same number of variables and disjunction in an irredundant d.n.f.. They suggested an algorithm for testing if a monotone Boolean function with $n=k$ is self-dual. Time complexity of this algorithm is $O\left(n^{4}\right)$.

In this paper we prove that interval graph of a monotone Boolean function is complete. It follows that the abbreviated d.n.f. of this function is also a minimal d.n.f. and a shortest d.n.f.. Then we present a d.n.f. with the highest number of conjunctions and we determinate the exact number of them. We count the number of monotone Boolean functions whose maximal intervals have special properties. In the end we show upper and lower bounds for the number of Boolean function that are monotone and self-dual at the same time.

\section{PRELIMINARIES AND NOTATION}

We use the standard notation from Boolean function theory. An $n-$ ary Boolean function is a function $f:\{0,1\}^{n} \rightarrow\{0,1\}$.

Monotone Boolean function is a Boolean function $f\left(x_{1}, \ldots, x_{n}\right)$, having the following property: If for some sets $\tilde{\alpha}=\left(\alpha_{1}, \ldots, \alpha_{n}\right)$ and $\tilde{\beta}=\left(\beta_{1}, \ldots, \beta_{n}\right)$, where $\alpha_{i}, \beta_{i} \in\{0,1\}$, the condition $\alpha_{i} \leq \beta_{i}$ holds for all $1 \leq i \leq n$, then $f(\tilde{\alpha}) \leq f(\tilde{\beta})$. 
Self-dual Boolean function is a Boolean function $f\left(x_{1}, \ldots, x_{n}\right)$, having the following property: If for some sets $\tilde{\alpha}=\left(\alpha_{1}, \ldots, \alpha_{n}\right)$ and $\tilde{\beta}=\left(\beta_{1}, \ldots, \beta_{n}\right)$, where $\alpha_{i}, \beta_{i} \in\{0,1\}$, the condition $\alpha_{i}=\neg \beta_{i}$ holds for all $1 \leq i \leq n$, then $f(\tilde{\alpha})=\neg f(\tilde{\beta})$.

Boolean function is a symmetric Boolean function, if there exists a set $\left\{P_{1}, \ldots, P_{k}\right\}$, where $1 \leq k \leq n$ and $P_{i} \in\{0,1, \ldots, n\}$ while the function gains value 1 in the vertices which contain value 1 on $P_{i}$ positions. We denote level of Boolean cube $B^{n}$ as $P_{i}$ and symmetric Boolean function with levels $P_{1}, \ldots, P_{k}$ as $B_{P_{1}, \ldots, P_{k}}^{n}$.

The symbol $P^{n}$ denotes the set of all $n$-ary Boolean functions. Boolean variables and their negations are called literals. A literal of a variable $x$ is denoted by $x^{\alpha}$, where $\alpha \in\{0,1\}$, and we set

$$
x^{\alpha}=\left\{\begin{array}{l}
\bar{x}, \text { if } \alpha=0 \\
x, \text { if } \alpha=1
\end{array}\right.
$$

A conjunction $K=x_{i_{1}}^{\alpha_{i_{1}}} \wedge \ldots \wedge x_{i_{r}}^{\alpha_{i_{r}}}$ of literals of different variables is called an elementary conjunction. The number of literals $(r)$ in $K$ is called rank of $K$. A special case is the conjunction of rank 0 , it is called empty and its value is set to 1 .

A formula $D=K_{1} \vee \ldots \vee K_{s}$, the disjunction of distinct elementary conjunction, is called a disjunctive normal form. The parameter $s$ (the number of elementary conjunctions in $D$ ) is called the length of $D$. A d.n.f. with $s=0$ is called empty and its value is set to 0 . A d.n.f. $D$ represents a Boolean function $f$ if the truth tables of $f$ and $D$ coincide. Let us consider the class of all d.n.f. that represent an $n-$ ary Boolean function $f$. A d.n.f. with minimal number of literals in this class is called a minimal d.n.f. of $f$ and the one with minimal length in this class is called a shortest d.n.f. of $f$.

We also use a geometric representation of Boolean functions. The Boolean $n$-cube is the graph $B^{n}$ with $2^{n}$ vertices $\tilde{\alpha}=\left(\alpha_{1}, \ldots, \alpha_{n}\right)$, where $\alpha_{i} \in\{0,1\}$, in which those pairs of vertices that differ in exactly one coordinate are joined with an edge. For an $n$-ary Boolean function $f$ let $N_{f}$ denote the subset $\{\tilde{\alpha} ; f(\tilde{\alpha})=1\}$ and $N_{f}^{-}$denote the subset $\{\tilde{\alpha} ; f(\tilde{\alpha})=0\}$ of all vertices $\tilde{\alpha}$. Notice that there is a one-to-one correspondence between the sets $N_{f}$ and Boolean functions $f$. The 
subgraph of the Boolean $n$-cube induced by the set $N_{f}$ is called the graph of $f$ and is denoted by $G(f)$.

The set of vertices $N_{i} \subseteq\{0,1\}^{n}$ corresponding to an elementary conjunction $K_{i}$ of rank $r$ is called the interval of rank $r$. Notice that to every elementary conjunction $K=x_{i_{1}}^{\alpha_{i_{1}}} \wedge \ldots \wedge x_{i_{r}}^{\alpha_{i_{r}}}$ there corresponds an interval of rank $\mathrm{r}$ consisting of all vertices $\left(\beta_{1}, \ldots, \beta_{n}\right)$ of $B^{n}$ such that $\beta_{i_{j}}=\alpha_{i_{j}}$ for $j=1, \ldots, r$ and values of other vertex coordinates are arbitrary. In the present paper, we often work with intervals corresponding to elementary conjunctions.

In the geometric model, every interval of rank $r$ represents an $(n-$ $r$ )-di-mensional subcube of $B^{n}$. Therefore we call the interval of rank $r$ also the $(n-r)$-dimensional interval. An interval $N$ is called the maximal interval of Boolean function $f$ if $N \subseteq N_{f}$ and there is no interval $N^{\prime} \subseteq N_{f}$ such that $N \subseteq N^{\prime}$. A d.n.f. which consists of all elementary conjunctions corresponding to maximal intervals is called the abbreviated d.n.f. and it is denoted by $D_{A}(f)$.

Now we can define the interval graph $\Gamma(f)$ as the graph associated with a Boolean function $f$ as follows: its vertices correspond to maximal intervals of $f$ and the vertices corresponding to intervals $N_{i}$ and $N_{j}$ are joined with an edge in $\Gamma(f)$ if $K_{i} \wedge K_{j}$ is nonempty.

For an arbitrary Boolean function $f$ and each of its d.n.f.s $K_{1} \vee \ldots \vee K_{s}$, we have that

$$
N_{f}=\bigcup_{j=1}^{s} N_{j} .
$$

In other words, every d.n.f. of a Boolean function $f$ corresponds to a covering of $N_{f}$ by intervals $N_{1}, \ldots, N_{s}$ such that $N_{j} \subseteq N_{f}$. Conversely, every covering of $N_{f}$ by intervals $N_{1}, \ldots, N_{s}$ contained in $N_{f}$ corresponds to some d.n.f. of $f$. Using the geometric interpretation of d.n.f.s, we can express the irreducibility of d.n.f.. The d.n.f. $D$ of a Boolean function $f$ cannot be simplified if every interval $N_{j}$ of the covering corresponding to $D$ contains at least one vertex belonging to just this one interval of the covering. Such a d.n.f. is called an irredundant d.n.f.. 
Let $r_{j}$ denote the order of the interval $N_{j}$. Then the number of literals in d.n.f. is $r=\sum_{j=1}^{s} r_{j}$ and the construction of a minimal d.n.f. in the geometric model can be formulated as a problem of constructing a covering of $N_{f}$ by intervals $N_{j} \subseteq N_{f}$ with minimal $r$. On the other hand, the construction of a covering corresponding to the shortest d.n.f. requires to minimize the number of intervals in a covering of $N_{f}$.

\section{THE HIGHEST NUMBER OF CONJUNCTIONS}

In this section we study the properties of monotone Boolean function. First we prove the following result: the interval graph of a monotone Boolean function is complete. It follows that the abbreviated d.n.f. of this function is also a minimal d.n.f. and a shortest d.n.f.. Then we present a d.n.f. with the highest number of conjunctions and we determinate the exact number of them. We count the number of monotone Boolean functions whose maximal intervals have special properties which we describe later. In the end we show upper and lower bounds for the number of Boolean function that are monotone and self-dual at the same time.

LEMMA 3.1. The interval graph $\Gamma(f)$ of a monotone Boolean function $f$ is complete.

PROOF. Let $f$ be a Boolean function. Let $\delta$ be an arbitrary vertex such that $\delta \in N_{f}$ and $\delta \in P_{j}$. Then $\delta$ contains $n-j$ zero coordinates $x_{i_{1}}, \ldots, x_{i_{n-j}}$. We create $\delta^{\prime}$ by negation of a coordinate $x_{i_{k}}$ for all $1 \leq k \leq(n-j)$. Function $f$ is monotone and $\delta \leq \delta^{\prime}$, therefore $\delta^{\prime} \in N_{f}$. It implies that all vertices on the shortest path $(\delta, \underbrace{(1, \ldots, 1)}_{n})$ belong to the set $N_{f}$. Hence, all maximal intervals have nonempty intersection - the vertex $\underbrace{(1, \ldots, 1)}_{n}$. So any two arbitrary vertices of $\Gamma(f)$ are joined with an edge.

As a result we get that the abbreviated d.n.f. of a monotone Boolean function is also a minimal d.n.f. and a shortest d.n.f.. 
We determinate the exact number of conjunction in a d.n.f. of a monotone Boolean function that contains the highest number of them.

\section{THEOREM 3.2. Let $f$ be a monotone Boolean function of $n$ variables} containing the highest number of conjunctions in $D_{A}(f)$ (the highest number of maximal intervals in $\left.B^{n}\right)$. Then number of conjunctions is $\left(\begin{array}{c}n \\ {[n / 2]}\end{array}\right)$.

PROOF. Let $f$ be an arbitrary monotone Boolean function of $n$ variables with $k$ maximal intervals.

It follows from Theorem 3.1. that the interval graph of a monotone Boolean function is complete. This, together with definition of monotone Boolean function, implies a nonempty intersection of all maximal intervals - the vertex $\underbrace{(1, \ldots, 1)}_{n}$.

Let $l_{1}, \ldots, l_{k}$ such that $l_{1} \leq l_{2} \leq \cdots \leq l_{k}$ be levels $\alpha_{1}, \ldots, \alpha_{k}$ of the vertices of maximal intervals that are furthermost from vertex $\underbrace{(1, \ldots, 1)}_{n}$ of a Boolean function $f$. We call these vertices terminal. Let the level $l_{i}$ correspond to the vertex $\alpha_{i}$ and vice versa.

We prove that after finite number of steps we can construct a monotone Boolean function containing more maximal intervals with the same dimension - such that terminal vertices of maximal intervals are on the same level.

We describe two operations: an operation of movement towards the vertex $\underbrace{(1, \ldots, 1)}_{n}$ and an operation of movement towards the vertex $\underbrace{(0, \ldots, 0)}_{n}$. We show that under certain circumstances these operations increase the number of maximal intervals.

Let $l_{1}, \ldots, l_{s}$ for $1 \leq s \leq k$ and $s \leq\left(\begin{array}{l}n \\ l_{1}\end{array}\right)$, and $0 \leq l_{i}<\left[\frac{\mathrm{n}}{2}\right]$, where $1 \leq i \leq s$ have the same value. Each $\alpha_{i} \in\left\{\alpha_{1}, \ldots, \alpha_{s}\right\}$ is joined with $n-l_{1}$ vertices $\beta_{i_{1}}, \ldots, \beta_{i_{n-l_{1}}}$ on the level $l_{1}+1$. The sets of these vertices have a nonempty intersection if and only if the distance between the pairs of terminal vertices $\alpha_{i_{1}}$ and $\alpha_{i_{2}}$ for $1 \leq i_{1}<i_{2} \leq s$ is 2 (one step to the level $l_{1}+1$ and one back). Therefore, we replace maximal intervals containing terminal vertices $\alpha_{1}, \ldots, \alpha_{s}$ of dimension 
$n-l_{1}$ with at least $\frac{s \cdot\left(n-l_{1}\right)}{\left(l_{1}+1\right)}$ maximal intervals of dimension $n-\left(l_{1}+1\right)$ (where $l_{1}+1$ is the number of edges joining terminal vertex with vertices on actual level). It is easy to see that

$$
\text { (*) } \frac{s \cdot\left(n-l_{1}\right)}{\left(l_{1}+1\right)}>s \text {, }
$$

for even $n$ and for $0 \leq l_{1}<[\mathrm{n} / 2]-1$ where $n$ is odd. It follows that we increased the number of maximal intervals. For $l_{1}=[\mathrm{n} / 2]-1$ and $n$ odd we do not decrease number of maximal intervals (we also make this step). We replace the values $l_{1}, \ldots, l_{s}$ with new values.

We call this process movement towards the vertex $\underbrace{(1, \ldots, 1)}_{n}$ and we repeat it until $l_{1}<$ [n/2] holds. So while $n-l_{1} \leq l_{1}$ the number of edges from the vertex $\alpha_{1}$ to level $l_{1}+1$ is greater than (for $n$ odd in the last step equal to) the number of edges to the level $l_{1}-1$. In other words, while we increase the number of maximal intervals (or not decrease).

Now we describe the second operation - movement towards the vertex $\underbrace{(0, \ldots, 0)}_{n}$.

Let $l_{1} \geq \cdots \geq l_{j}$ be the levels (permuted) created in the movement towards the $\operatorname{vertex} \underbrace{(1, \ldots, 1)}_{n}$.

Let $l_{1}, \leq, l_{s}$ for $1 \leq s \leq k$ and $s \leq n_{l_{1}}$ and $[\mathrm{n} / 2]<l_{i} \leq n$, where $1 \leq i \leq$ $s$ have the same value. Each $\alpha_{i} \in\left\{\alpha_{1}, \ldots, \alpha_{s}\right\}$ is joined with $l_{1}$ vertices $\beta_{i_{1}}, \ldots, \beta_{i_{l_{1}}}$ on the level $l_{1}-1$. The sets of these vertices do not belong to any maximal intervals (otherwise the interval containing terminal vertex $\alpha_{i}$ would not be maximal) and have a nonempty intersection if and only if the distance between the pairs of terminal vertices $\alpha_{i_{1}}$ and $\alpha_{i_{2}}$ for $1 \leq i_{1}<i_{2} \leq s$ is 2 (one step to the level $l_{1}-1$ and one back). Therefore, we can replace maximal intervals containing terminal vertices $\alpha_{1}, \ldots, \alpha_{s}$ of dimension $n-l_{1}$ by at least $\frac{s . l_{1}}{n-\left(l_{1}-1\right)}$ maximal intervals of dimension $n-\left(l_{1}-1\right)$ (where $n-\left(l_{1}-1\right)$ is the number of edges joining terminal vertex 
with vertices on actual level). By increasing the dimension of maximal intervals can happened that we include the vertices on level $l_{1}-1$. These vertices belong to one of the sets $N_{f}$ (intervals, whose terminal vertices are on lower level or) $N_{f}^{-}$. It is easy to see that

$$
(* *) \quad \frac{s . l_{1}}{n-\left(l_{1}-1\right)}>s .
$$

It follows that we increased the number of maximal intervals. We replace the values $l_{1}, \ldots, l_{s}$ with new values.

We repeat the movement towards the vertex $\underbrace{(0, \ldots, 0)}_{n}$ until $[\mathrm{n} / 2]<l_{1}$ holds. In other words, until we cannot increased the number of maximal intervals.

Let $\alpha_{i} \in\left\{\alpha_{1}, \ldots, \alpha_{\}}\right.$. As $\alpha_{i}$ is a terminal vertex, there is no vertex from the set $\left\{\alpha_{1}, \ldots, \alpha_{k}\right\} /\left\{\alpha_{i}\right\}$ on any shortest path $(\alpha_{i}, \ldots, \underbrace{(0, \ldots, 0)}_{n})$ or $(\alpha_{i}, \ldots, \underbrace{(1, \ldots, 1)}_{n})$. It follows that we can perform operations of movement for each level independently and terminal vertices on other levels are not affected. It also implies that the distance between terminal vertices on different levels is at least 3 .

From Pascal Triangle and inequalities $(*)$ and $(* *)$ we know that the highest number of vertices is on the level $n / 2$ for $n$ even and on the levels [n/2] and [n/2] for $n$ odd. Therefore if we apply this process on input set of terminal vertices we reach this level. The number of terminal vertices (and thus the number of maximal intervals) is $\left(\begin{array}{c}n \\ {[n / 2]}\end{array}\right)$.

It is easy to see that aplication of these operations does not violate property of monotonicity.

In Figures 1. we illustrate the process described in Proof of Theorem 3.2.. Let $f$ be a monotone Boolean function of 5 variables for wich $\left|D_{A}(f)\right|=4$ holds and $D_{A}(f)=x_{2} x_{3} x_{4} x_{5} \vee x_{1} x_{4} x_{5} \vee x_{1} x_{3} \vee x_{1} x_{2}$. Function $f$ corresponds to $B^{5}$ in the figures. 


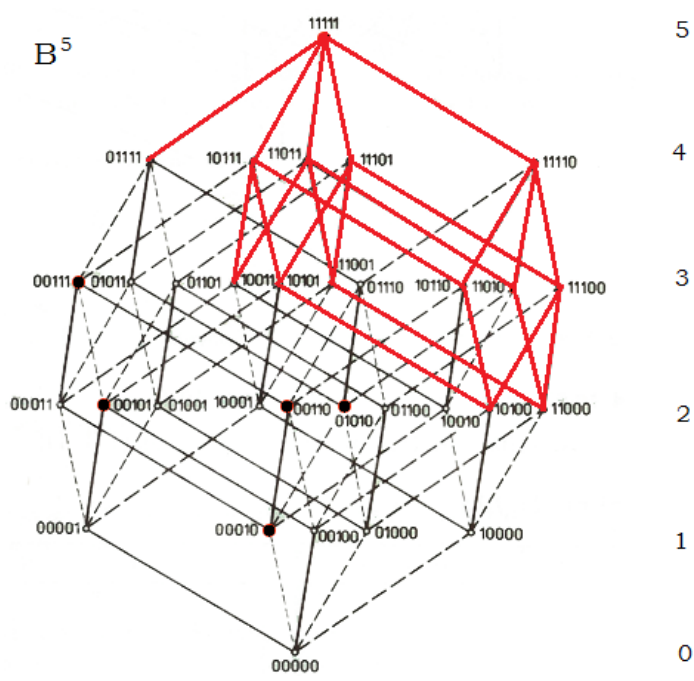

Figure $1: k=4, l_{1}=l_{2}=2, l_{3}=3, l_{4}=4, s=2, \alpha_{1}=(1,0,1,0,0), \alpha_{2}=(1,1,0,0,0), \alpha_{3}=$ $(1,0,0,1,1), \alpha_{4}=(0,1,1,1,1)$

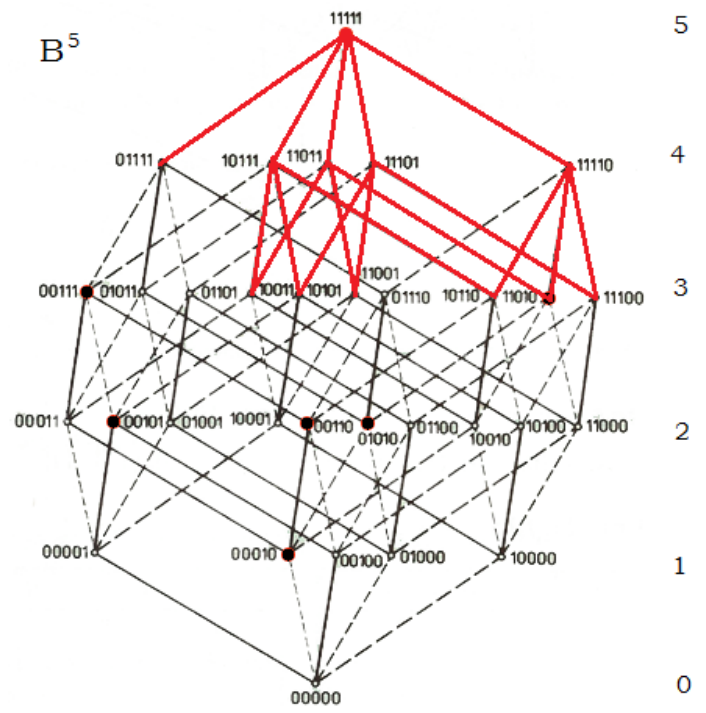

$$
\begin{gathered}
k=7, l_{1}=4, l_{2}=l_{7}=3, s=1, \alpha_{1}=(0,1,1,1,1), \alpha_{2}=(1,0,0,1,1), \alpha_{3}=(1,0,1,0,1), \\
\alpha_{4}=(1,1,0,0,1), \alpha_{5}=(1,0,1,1,0), \alpha_{6}=(1,1,0,1,0), \alpha_{7}=(1,1,1,0,0)
\end{gathered}
$$




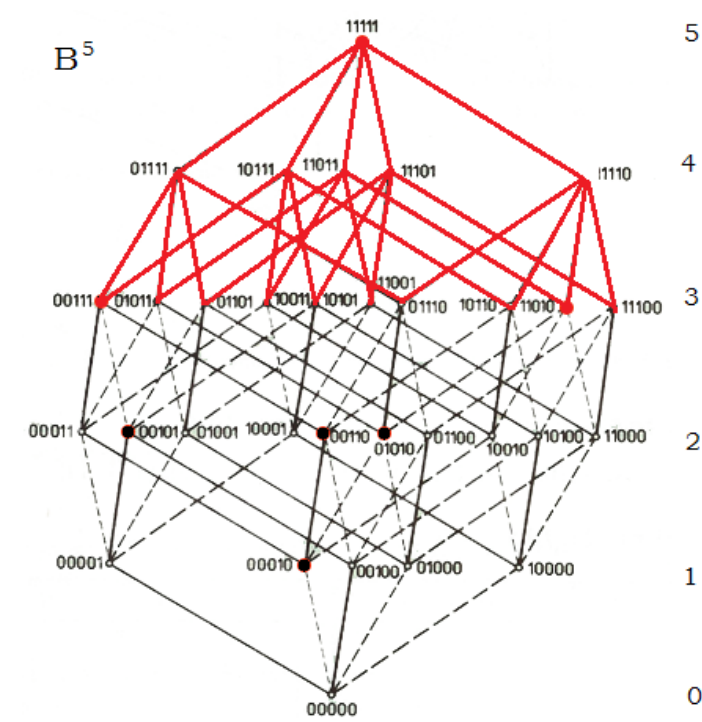

$$
k=10=\left(\begin{array}{c}
n \\
{[n / 2]}
\end{array}\right), l_{1}=l_{10}=3, s=10, \alpha_{1}=(0,0,1,1,1), \alpha_{2}=(0,1,0,1,1), \alpha_{3}=
$$

$(0,1,1,0,1), \alpha_{4}=(1,0,0,1,1), \alpha_{5}=(1,0,1,0,1), \alpha_{6}=(1,1,0,0,1), \alpha_{7}=(0,1,1,1,0), \alpha_{8}=(1,0,1,1,0)$,

$$
\alpha_{9}=(1,1,0,1,0), \alpha_{10}=(1,1,1,0,0)
$$

\section{THE NUMBER OF MONOTONE BOOLEAN FUNCTIONS WITH SPECIAL PROPERTIES OF MAXIMAL INTERVALS}

THEOREM 4.1. Let $G=(V, E)$ be a complete graph of order $n$. Let $f$ be a monotone Boolean function of $n$ variables whose maximal intervals have the same dimension and $\Gamma(f) \cong G$. Then the number of such functions is

where $m \leq\left(\begin{array}{c}n \\ {[n / 2]}\end{array}\right)$

$$
\left.\sum_{i=0}^{n}\left(\begin{array}{c}
n \\
i \\
m
\end{array}\right)\right)
$$

PROOF. Let $G=(V, E)$ be a complete graph of order $m$. Let $f$ be a monotone Boolean function of $n$ variables whose maximal intervals have the same dimension and for which $\Gamma(f) \cong G$ holds. Since $G$ is of order $m, \Gamma(f)$ is of order $m$ and number of maximal intervals of function $f$ is $m$. As they have the same dimension, the terminal vertices would be on the same level. Terminal vertex on level $i$ represents a maximal interval of dimension $n-i$. It follows that the number of 
monotone Boolean functions is

$$
\left.\sum_{i=0}^{n}\left(\begin{array}{c}
n \\
i \\
m
\end{array}\right)\right)
$$

THEOREM 4.2. Let $G=(V, E)$ be a complete graph of order $m$. Then the number of monotone Boolean functions of $n$ variables in which the intersection of an arbitrary pair of maximal intervals is only vertex $\underbrace{(1, \ldots, 1)}_{n}$ and for which $\Gamma(f) \cong G$ holds is

$$
\frac{\sum_{i=0}^{m}(-1)^{i} \cdot\left(\begin{array}{c}
m \\
i
\end{array}\right) \cdot(m+1-i)^{n}}{m !}
$$

where $1 \leq m \leq n-1$.

PROOF. Let $G=(V, E)$ be a complete graph of order $m$. Let $f$ be a monotone Boolean function of $n$ variables such that the intersection of an arbitrary pair of maximal intervals is only vertex $\underbrace{(1, \ldots, 1)}_{n}$ and for which $\Gamma(f) \cong G$ holds. Since $G$ is of order $m, \Gamma(f)$ is of order $m$ and number of maximal intervals of function $f$ is $m$. Let $N_{1}, \ldots, N_{m}$ be maximal intervals corresponding to $m$ vertices of the graph $\Gamma(f)$. Vertex $\underbrace{(1, \ldots, 1)}_{n}$ is of degree $n$. For each of its $n$ edges we have $m+1$ possibilities: either it belongs to maximal interval $N_{1}$ (we denote this option as 1 ), ..., or it belongs to maximal interval $N_{m}$ (we denote this option as $m$ ), or it do not belong to any maximal interval (we denote this option as 0$)$. Vector $\left(\gamma_{1}, \ldots, \gamma_{n}\right)$, where $\gamma_{i} \in(0, \ldots, m)$ clearly identifies the location and dimension of $m$ maximal intervals. All the values $1, \ldots, m$ have to be in the vector to keep the number of maximal intervals. We subtract the unsufficient options from $(m+1)^{n}$. We use the inclusion-exclusion principle to make sure that each $n$-tuple is counted exactly once. Since we do not care about the order of maximal intervals, we divide the final sum with the number of permutations of $m$ maximal intervals. It follows that the number of monotone Boolean functions is 


$$
\frac{\sum_{i=0}^{m}(-1)^{i} \cdot\left(\begin{array}{c}
m \\
i
\end{array}\right) \cdot(m+1-i)^{n}}{m !} .
$$

Now we estimate the expression from the last Theorem.

THEOREM 4.3.

$$
\begin{aligned}
& \frac{1}{2}\left((m+1)^{2}+m+3\right)(m+1)^{n-m-1}-1 \leq \\
& \leq \frac{\sum_{i=0}^{m}(-1)^{i} \cdot\left(\begin{array}{c}
m \\
i
\end{array}\right) \cdot(m+1-i)^{n}}{m !} \leq \frac{1}{2}\left(\begin{array}{c}
n+1 \\
m+1
\end{array}\right)(m+1)^{n-m},
\end{aligned}
$$

where $n \leq 2$ and $1 \leq m \leq n-1$.

PROOF. We rewrite the following expression as

$$
\begin{aligned}
& \frac{\sum_{i=0}^{m}(-1)^{i} \cdot\left(\begin{array}{c}
m \\
i
\end{array}\right) \cdot(m+1-i)^{n}}{m !}= \\
& =\frac{\sum_{i=0}^{m}(-1)^{m-i} \cdot\left(\begin{array}{c}
m \\
i
\end{array}\right) \cdot(i+1)^{n}}{m !}= \\
& =\frac{1}{m !} \sum_{i=0}^{m}(-1)^{m-i} \cdot\left(\begin{array}{c}
m \\
i
\end{array}\right) \cdot \sum_{k=0}^{n}\left(\begin{array}{l}
n \\
k
\end{array}\right) i^{k}= \\
& =\sum_{k=0}^{n}\left(\begin{array}{l}
n \\
k
\end{array}\right) \frac{\sum_{i=0}^{m}(-1)^{m-i} \cdot\left(\begin{array}{c}
m \\
i
\end{array}\right) \cdot i^{k}}{m !}
\end{aligned}
$$

Using Knuth 6.19 from [11] we get

$$
\sum_{k=0}^{n}\left(\begin{array}{l}
n \\
k
\end{array}\right) \frac{\sum_{i=0}^{m}(-1)^{m-i} \cdot\left(\begin{array}{c}
m \\
i
\end{array}\right) \cdot i^{k}}{m !}=\sum_{k=0}^{n}\left(\begin{array}{l}
n \\
k
\end{array}\right)\left\{\begin{array}{l}
k \\
m
\end{array}\right\}
$$

Aplying Knuth 6.15 from [11] we get

$$
\sum_{k=0}^{n}\left(\begin{array}{l}
n \\
k
\end{array}\right)\left\{\begin{array}{l}
k \\
m
\end{array}\right\}=\left\{\begin{array}{c}
n+1 \\
m+1
\end{array}\right\} .
$$

Asymptotic Estimates of Stierling numbers is known

$$
\frac{1}{2}\left((m+1)^{2}+m+3\right)(m+1)^{n-m-1}-1 \leq\left\{\begin{array}{c}
k \\
m
\end{array}\right\} \leq \frac{1}{2}\left(\begin{array}{c}
n+1 \\
m+1
\end{array}\right)(m+1)^{n-m},
$$

for $n \geq 2$ and $1 \leq m \leq n-1$. 
In this part we show upper and lower bound for the number of Boolean functions that are monotone and self-dual at the same time.

\section{BOUNDS}

THEOREM 5.1. The number of monotone and at the same time self-dual Boolean functions of $n$ variables is at most

$$
1+\sum_{l=1}^{\left[\frac{n}{2}\right]} \sum_{i=1}^{\left(\begin{array}{c}
n-1 \\
l-1
\end{array}\right)}\left(\left(\begin{array}{c}
n \\
n-l \\
i
\end{array}\right)\right)
$$

for $n$ odd and

$$
2^{\frac{\left(\begin{array}{c}
n \\
n / 2
\end{array}\right)}{2}}+\sum_{l=1}^{\frac{n}{2}-1} \sum_{i=1}^{\left(\begin{array}{c}
n-1 \\
l-1
\end{array}\right)}\left(\left(\begin{array}{c}
n \\
n-l \\
i
\end{array}\right)\right)
$$

for $n$ even.

PROOF. Let $f$ be a monotone and at the same time self-dual Boolean function of $n$ variables. Monotony implies that all intervals contain the vertex $\underbrace{(1, \ldots, 1)}_{n}$. Therefore $f(\underbrace{(1, \ldots, 1)}_{n})=1$. Self-duality implies that $\left|N_{f}\right|=2^{n-1}$ and $\left|N_{f}^{-}\right|=$ $2^{n-1}$

We divide the proof in to two parts. In the first part we assume that $n$ is odd. Now we estimate the maximal number of intervals which can be on particular levels such that function $f$ is self-dual.

It is easy to see that every vertex on levels $\left[\frac{n}{2}\right], \ldots, n$ can be terminal. There can be at most $\left(\begin{array}{c}n-1 \\ l-1\end{array}\right)$ terminal vertices on levels $l \in\left\{1, \ldots,\left[\frac{n}{2}\right]\right\}$. This number of vertices can be obtained, if we take the largest maximal interval such that the function is monotone and at the same time self-dual, interval of dimension $n-1$ containing $2^{n-1}$ vertices, containing vertex $\underbrace{(1, \ldots, 1)}_{n}$ (the levels are moved). We do not add the terminal vertex on any levels, because it contradicts with $\left|N_{f}\right|>2^{n-1}$. For the vertex 
$\underbrace{(0, \ldots, 0)}_{n}$ on level $0 f(\underbrace{(0, \ldots, 0)}_{n})=0$ holds.

Self-duality also implies that for every vertex $\alpha$ the following holds: If $f(\alpha)=x$, then $f(\neg \alpha)=\neg x$ and if $\alpha$ is situated on a level from $0, \ldots,\left[\frac{n}{2}\right]$, then $\neg \alpha$ is situated on a level from $\left[\frac{n}{2}\right], \ldots, n$ and vice versa. The sets $\left|N_{f}\right|$ and $\left|N_{f}^{-}\right|$are clearly identified by the selection of terminal vertices on levels $1, \ldots,\left[\frac{n}{2}\right]$ opposite vetrices to terminal vertices are in the set $\left|N_{f}^{-}\right|$, all vertices on the shortest path from these vertices to vertex $\underbrace{(0, \ldots, 0)}_{n}$ are in the set $\left|N_{f}^{-}\right|$(these verices are opposite to vertices of maximal intervals generated by terminal vertices), other vertices on levels $\left[\frac{n}{2}\right], \ldots, n$ are in the set $\left|N_{f}\right|$ (these vertices are opposite to vertices taking value 0 situated on levels $1, \ldots,\left[\frac{n}{2}\right]$ ). This distribution does not except the creation of new terminal vertices on levels $\left[\frac{n}{2}\right], \ldots, n$. It is obvious that this distribution is symmetric and $\left|N_{f}\right|=\left|N_{f}^{-}\right|=2^{n-1}$ holds.

This distribution to the sets $\left|N_{f}\right|$ and $\left|N_{f}^{-}\right|$does not exist for every selection of terminal vertices. Therefore we count the function which are not self-dual by selection of terminal vertices on the levels $1, \ldots,\left[\frac{n}{2}\right]$ It is easy to see that we do not violate monotonicity.

We have to add the situation, where all terminal vertices are on the level $\left[\frac{n}{2}\right]$. It follows that the number of monotone and at the same time self-dual Boolean function $f\left(x_{1}, \ldots, x_{n}\right)$ for $n$ odd is at most

$$
1+\sum_{l=1}^{\left[\frac{n}{2}\right]} \sum_{i=1}^{\left(\begin{array}{c}
n-1 \\
l-1
\end{array}\right)}\left(\left(\begin{array}{c}
n \\
n-l \\
i
\end{array}\right)\right)
$$

In the second part we assume that $n$ is even. Then $f$ has odd number of levels. We obtain the upper bound analogically as in the first part of proof - we count the possibilities for levels $1, \ldots, \frac{n}{2}-1$. Self-duality implies that $\frac{\left(\begin{array}{c}n \\ n / 2\end{array}\right)}{2}$ vertices on level 
$n / 2$ belong to the set $\left|N_{f}\right|$ and $\frac{\left(\begin{array}{c}n \\ n / 2\end{array}\right)}{2}$ vertices belongs to the set $\left|N_{f}^{-}\right|$. We add this possibility to the sum.

It follows that the number of monotone and at the same time self-dual Boolean function $f\left(x_{1}, \ldots, x_{n}\right)$ for $n$ even is at most

$$
2^{\frac{\left(\begin{array}{c}
n \\
n / 2
\end{array}\right)}{2}}+\sum_{l=1}^{\frac{n}{2}-1} \sum_{i=1}^{\left(\begin{array}{c}
n-1 \\
l-1
\end{array}\right)}\left(\left(\begin{array}{c}
n \\
n-l \\
i
\end{array}\right)\right) .
$$

REMARK 5.2. All of Boolean functions counted in the sum are monotone, but not all of them are necessarily self-dual.

THEOREM 5.3. The upper bound of monotone and at the same time self-dual boolean function of $n$ variables is asymptoticly equal to

for $n$ even and

$$
\frac{2^{\left(\begin{array}{c}
n \\
n / 2-1
\end{array}\right)}}{\sqrt{\left(\begin{array}{c}
n \\
n / 2-1
\end{array}\right)}} \cdot 2^{O(1)},
$$

for $n$ odd.

$$
\frac{2^{\left(\begin{array}{c}
n \\
{[n / 2]}
\end{array}\right)}}{\sqrt{\left(\begin{array}{c}
n \\
{[n / 2]}
\end{array}\right)}} \cdot 2^{O(1)}
$$

PROOF. The number of monotone and at the same time self-dual Boolean function of $n$ variables for $n$ even is at most

$$
\begin{gathered}
2^{\frac{\left(\begin{array}{c}
n \\
n / 2
\end{array}\right)}{2}}+\sum_{l=1}^{\frac{n}{2}-1} \sum_{i=1}^{\left(\begin{array}{c}
n-1 \\
l-1
\end{array}\right)}\left(\left(\begin{array}{c}
n \\
n-l \\
i
\end{array}\right)\right)= \\
2^{\frac{\left(\begin{array}{c}
n \\
n / 2
\end{array}\right)}{2}}+\sum_{l=1}^{\frac{n}{2}-1 \frac{1}{n}\left(\begin{array}{c}
n \\
l
\end{array}\right)} \sum_{i=1}^{(}\left(\begin{array}{c}
n \\
l \\
i
\end{array}\right)
\end{gathered}
$$

Using the estimate

$$
\sum_{k \leq \alpha . n}\left(\begin{array}{l}
n \\
k
\end{array}\right)=2^{n \cdot H(\alpha)-\frac{1}{2} \cdot \log n+O(1)},
$$

where $0<\alpha<\frac{1}{2}$ and $H(\alpha)=\alpha \cdot \log \frac{1}{\alpha}+(1-\alpha) \cdot \log \frac{1}{1-\alpha}$, from [11] we get 


$$
2^{\frac{\left(\begin{array}{c}
n \\
n / 2
\end{array}\right)}{2}}+\sum_{l=1}^{\frac{n}{2}-1} 2\left(\begin{array}{l}
n \\
l
\end{array}\right) \cdot H\left(\frac{l}{n}\right)-\frac{1}{2} \cdot \log \left(\begin{array}{l}
n \\
l
\end{array}\right)+O(1)
$$

While the biggest summand is for $l=\frac{n}{2}-1$ and

$$
\begin{gathered}
\frac{n}{2}-1<\left(\begin{array}{c}
n \\
n / 2-1
\end{array}\right) \cdot H\left(\frac{n / 2-1}{n}\right)-\frac{1}{2} \cdot \log \left(\begin{array}{c}
n \\
n / 2-1
\end{array}\right)+O(1), \text { it holds that } \\
2^{\frac{\left(\begin{array}{c}
n \\
n / 2
\end{array}\right)}{2}}+\sum_{l=1}^{i} 2^{l}
\end{gathered}
$$

where $i=\left(\begin{array}{c}n \\ n / 2-1\end{array}\right) \cdot H\left(\frac{n / 2-1}{n}\right)-\frac{1}{2} \cdot \log \left(\begin{array}{c}n \\ n / 2-1\end{array}\right)+O(1)$

Using $\sum_{i=0}^{n} 2^{i}=2^{n+1}-1$ we get

$$
2^{\frac{\left(\begin{array}{c}
n \\
n / 2
\end{array}\right)}{2}}+2^{\left(\begin{array}{c}
n \\
n / 2-1
\end{array}\right) \cdot H\left(\frac{n / 2-1}{n}\right)-\frac{1}{2} \cdot \log \left(\begin{array}{c}
n \\
n / 2-1
\end{array}\right)+O(1)}-1 .
$$

Limit of a function $H\left(\frac{n / 2-1}{n}\right)$ is equal to 1 for $n \rightarrow \infty$, therefore

$$
\frac{2^{\left(\begin{array}{c}
n \\
n / 2-1
\end{array}\right)}}{\sqrt{\left(\begin{array}{c}
n \\
n / 2-1
\end{array}\right)}} \cdot 2^{O(1)} .
$$

We obtain the asymptotic estimate for the number of monotone and at the same time self-dual Boolean function of $n$ variables for $n$ odd analogically.

REMARK 5.4. We can compare our asymptotic estimate of upper bound of the number of monotone and at the same time self-dual Boolean functions with the best solution so far, asymptotic estimate of number of the monotone Boolean functions by A. D. Korshunov in [21] (see Section 1). It is easy to see that the class of monotone and at the same time self-dual Boolean function is much smaller.

THEOREM 5.5. The number of monotone and at the same time self-dual Boolean function of $n$ variables, where $n$ is even is at least

$$
2^{\frac{\left(\begin{array}{c}
n \\
n / 2
\end{array}\right)}{2}}
$$


PROOF. Let $f$ be monotone and at the same time self-dual Boolean function of $n$ variables. The function $f$ has the odd number of levels. Self-duality implies that $\frac{\left(\begin{array}{c}n \\ n / 2\end{array}\right)}{2}$ vertices on level $n / 2$ belong to the set $\left|N_{f}\right|$ and $\frac{\left(\begin{array}{c}n \\ n / 2\end{array}\right)}{2}$ vertices belong to the set $\left|N_{f}^{-}\right|$. The number of possibilities of distribution of $\left(\begin{array}{c}n \\ n / 2\end{array}\right)$ vertices to the sets $\left|N_{f}\right|$ and $\left|N_{f}^{-}\right|$such that the function is self-dual and monotone is

$$
2^{\frac{\left(\begin{array}{c}
n \\
n / 2
\end{array}\right)}{2}}
$$

REMARK 5.6. It is easy to see, that number of self-dual Boolean functions of $n$ variables is $2^{2^{n-1}}$. The number of monotone and at the same time symmetric Boolean functions of $n$ variables is $n+1$.

\section{CONCLUSION}

In this paper we proved that interval graph of a monotone Boolean function is complete. It follows that the abbreviated d.n.f. of this function is also a minimal d.n.f. and a shortest d.n.f.. Then we have presented a d.n.f. with the highest number of conjunctions and we determinated the exact number of them. We have counted the number of monotone Boolean functions whose maximal intervals have special properties. In the end we showed upper and lower bound for the number of Boolean function that are monotone and self-dual at the same time.

\section{REFERENCES}

[1] Yablonskiy S. V., Introduction into discrete mathematics, Moscow, Nauka, 1979 (in Russian).

[2] Yablonskiy S. V. and Lupanov O. B., Discrete mathematics and Mathematical Problems of Cybernetics, Nauka, Moscow, 1974, (in Russian)

[3] Sapozhenko A. A., Disjunctive Normal Forms, Moscow University Press, Moscow, 1975 (in Russian).

[4] Sapozhenko A. A., Geometric structure of almost all Boolean, Problemy kibernetiky, Vol. 30, 1975 , 227-261, (in Russian)

[5] Zhuravlev J. I., Set theoretical methods in the algebra of logic, Problemy kibernetiki, Vol. 8, 1962, 5-44, (in Russian).

[6] Toman E., On the size of a neighbourhood of the first rank, Computers and Artificial Intelligence, Vol.12, No. 2, 1993, 123-130

[7] Daubner J. and Toman E., Vertex degree in the interval graph of a random Boolean function, Acta Mathematica Universitatis Comenianae, Vol. 79, No. 2, 2010, 151-164

[8] Toman E., Olejar D. and Stanek M., Average degree in the interval graph of a random Boolean function, Computing and Informatics, Vol. 27, 2008, 627-638 
[9] Daubner J. and Toman E., Neighbourhood of the constant order in the interval graph of a random Boolean functionFundamenta Informaticae, 2012, Vol. 122, 1-21

[10] Toman E. and Tomanova J., Some estimates of the complexity of disjunctive normal forms of a random Boolean function, Computers and Artificial Intelligence, Vol. 10, No. 4, 1991, 327-340

[11] Graham, Knuth, Patashnik, Concrete Mathematics a Foundation for Computer Science, Addison-Wesley Publishing Company, 1989

[12] Dedekind R., Über Zerlegungen von Zahlen durch ihre größten gemeinsamen Teiler, Gesammelte Werke, 1897, Vol. 2, 103-148

[13] Church R., Numerical analysis of certain free distributive structures, Duke Mathematical Journal, 1940, Vol. 6, 732-734

[14] Ward M., Note on the order of free distributive lattices, Bulletin of the American Mathematical Society, 1946, Vol. 52, 423

[15] Gilbert E. N., Lattice theoretic properties of frontal switching functions, J. Math. Physics, 1954, Vol. 33, 57-67

[16] Yamamoto K., Note on the order of free distributive lattices, Science Reports of the Kanazawa University, 1953, Vol. 2, No. 1, 5-6

[17] [17] Hansel G., Sur le nombre des fonctions boolĂ(ennes monotones de $n$ variables, C. R. Acad. Sci. Paris, 1966, Vol. 262, No. 20, 1088-1090 (French)

[18] Markowsky G., Combinatorial aspects of lattice theory with applications to the enumeration of free distributive lattices, Ph.D. Thesis, Harvard University, 1973

[19] Kleitman D., Markowsky G., On Dedekind's problem: the number of isotone Boolean functions. II, Transactions of the American Mathematical Society, 1975, Vol. 21, 373-390

[20] Yamamoto K., Logarithmic order of free distributive lattice, Journal of the Mathematical Society of Japan, 1954, Vol. 6, 343-353

[21] Korshunov, A. D., The number of monotone Boolean functions, Problemy Kibernetiki, 1981, Vol. 38, 5-108

[22] Kleitman D., On Dedekind's problem: the number of monotone Boolean functions, Proc. Amer Math. Soc., 1969, Vol. 21, 677-682

[23] Post E., Introduction to a general theory of elementary propositions, American Journal of Mathematics, 1921, Vol. 4, 163-185

[24] Altun M. and Riedel M. D., A Study on Monotone Self-dual Boolean Functions, SIAM Journal on Discrete Mathematics, 2012, 10 pages

[25] E.Sperner, Ein Satz über Untermengen einer endlichen Menge, Math.Z., 27(1928), pp. 544-548

[26] G. Grätzer, Lattice Theory: Foundation, Springer Basel AG, 2011

[27] Movsisyan Yu. M., Aslanyan V.A., A functional completeness theorem for De Morgan functions, Discrete Appl. Math., 2014, 162, 1-16

\author{
L. HAVIAROVA and E. TOMAN \\ Comenius University \\ Faculty of Mathematics, Physics and Informatics \\ Department of Computer Science \\ Mlynska dolina, 84248 Bratislava \\ Slovak Republic
}

